Rapid Reviews COVID-19

\title{
Review 1: "Evaluation of a Machine Learning Approach Utilizing Wearable Data for Prediction of SARS-CoV-2 Infection in Healthcare Workers"
}

Aaron Hudson ${ }^{1}$

${ }^{1}$ UC Berkeley

Published on: Jan 26, 2022

License: Creative Commons Attribution 4.0 International License (CC-BY 4.0). 


\section{RR:C19 Evidence Scale rating by reviewer:}

- Potentially informative. The main claims made are not strongly justified by the methods and data, but may yield some insight. The results and conclusions of the study may resemble those from the hypothetical ideal study, but there is substantial room for doubt. Decision-makers should consider this evidence only with a thorough understanding of its weaknesses, alongside other evidence and theory. Decisionmakers should not consider this actionable, unless the weaknesses are clearly understood and there is other theory and evidence to further support it.

$* * * * * * * * * * * * * * * * * * * * * * * * * * * * * * * * * * * * * * *$

\section{Review:}

The authors discuss the use of wearable device data for non-invasive prediction of SARS-CoV-2 infection. It has previously been shown that heart monitoring data collected from wearable devices is associated with infection. In this work, the authors develop a prediction model for positive COVID-19 diagnosis based on data collected from Apple Watches on heart rate variability and resting heart rate data.

The authors' prediction algorithm can effectively predict positive COVID-19, with sensitivity and specificity of $76 \%$ and $84 \%$, respectively, on a testing data set. The authors also show that HRV measurements are among the most important predictors in the algorithm, suggesting that HRV should be considered as a potentially useful predictor variable in further development of algorithms for predicting COVID-19 diagnosis.

The authors' description of the construction of their predictive model is in some ways unclear to me. For instance, it is not clear how the smoothed estimates of daily HRV mean, amplitude and acrophase were calculated. I also feel it is not sufficiently well explained what are the parameters of the COSINOR random effects model the authors estimate or how the estimated COSINOR model was used in development the prediction model. Additionally, it would be helpful if the authors provided more details explaining how their training and testing sets were constructed. In particular, I do not understand how independence between the training and testing sets was achieved. It is also unclear how the authors accounted for correlation between observations when constructing confidence intervals for summaries of model performance on the training and testing set. 
I also suspect that an improved prediction model could possibly be constructed, which predicts COVID-19 positivity for a subject at any given time point, based on the subject's history up until that time point. My reasoning is that whether a subject, on a given day, observes HRV or heart rate measurements that are far different from the population average may be less relevant for predicting COVID-19 positivity than whether the subject observes a change in HRV and heart rate measurements that is substantially different from what is typical for that particular subject. I suggest that the authors consider this approach in their future research.

In summary, the authors' conclusion that wearable device data can be used to predict COVID-19 infection appears to be justified. However, some aspects of the author's methodology could have been explained more clearly, and the manuscript would benefit from the inclusion of more details. 